\title{
Desenvolvimento e aplicação de metodologia por cromatografia em camada delgada para determinação do perfil de alcalóides oxindólicos pentacíclicos nas espécies sul-americanas do gênero Uncaria
}

\author{
Ligia M.M. Valente ${ }^{1 *}$, Flaviane F. Alves ${ }^{1}$, Giselle M. Bezerra ${ }^{1}$, Maria Beatriz S. Almeida ${ }^{2}$, \\ Sandra L. Rosario', José L.Mazzei', Luiz A. d'Avila', Antonio C. Siani' \\ ${ }^{1}$ Departamento de Quimica Orgânica, Instituto de Química, Universidade Federal do Rio de Janeiro, Centro de \\ Tecnologia, Bloco A, 21910-240, Rio de Janeiro, RJ, Brasil, \\ ${ }^{2}$ Instituto de Tecnologia em Fármacos, Fundação Oswaldo Cruz, R. Sizenando Nabuco 100, \\ 21041-250, Rio de Janeiro, RJ, Brasil, \\ ${ }^{3}$ Departamento de Processos Orgânicos, Escola de Química, Universidade Federal do Rio de Janeiro, Centro de \\ Tecnologia, Bloco E, 21910-240, Rio de Janeiro, RJ, Brasil
}

\begin{abstract}
RESUMO: O gênero Uncaria (Rubiaceae) é representado na América do Sul e Central por duas espécies: U. tomentosa (Willd.) DC. e U. guianensis (Aubl.) Gmel., conhecidas popularmente como unha-de-gato. Ambas são trepadeiras perenes, sendo empregadas na prevenção e cura de várias doenças. Nessas plantas são encontrados alcalóides oxindólicos e indólicos, triterpenos glicosilados, taninos e flavonóides. Seis alcalóides oxindólicos pentacíclicos, considerados seus marcadores: especiofilina, mitrafilina, uncarina $\mathrm{F}$, isomitrafilina, pteropodina e isopteropodina, são usados na padronização do material vegetal e fitoterápicos derivados. O presente trabalho descreve o desenvolvimento de metodologia analítica qualitativa utilizando cromatografia em camada delgada (CCD) para determinação do perfil dos seis alcalóides oxindólicos pentacíclicos marcadores das espécies. O desenvolvimento do método incluiu a comparação entre o uso do extrato metanólico bruto, e de frações enriquecidas obtidas por partição ácido-base clássica ou pelo uso de resina básica Poliamida 6. Utilizou-se gel de sílica como fase estacionária, e variaram-se alguns parâmetros como: eluentes, concentração da amostra, espaço de eluição e tipos de reveladores. O método desenvolvido em CCD mostrou-se confiável, reprodutível e seletivo para os alcalóides alvos, sendo aplicado na análise de amostras de folhas e caule das duas espécies e também de fitoterápicos comerciais à base de $U$. tomentosa.
\end{abstract}

Unitermos: Uncaria tomentosa, Uncaria guianensis, Rubiaceae, alcalóides oxindólicos, cromatografia em camada delgada.

\begin{abstract}
Development and application of a thin layer chromatographic method for the determination of the pentacyclic oxindole alkaloid profile in South-American species of the genus Uncaria". The species Uncaria tomentosa (Willd.) DC. and U. guianensis Gmel. (Rubiaceae), known as cat's claw, are large woody vines occurring in the Amazon rain forest and other tropical areas of South and Central America. It has been used medicinally by indigenous peoples for at least 2,000 years for several diseases. Tetra- and pentacyclic oxindole alkaloids, triterpenoid glycosides, sterols and flavonoids are found in these plants. Among these metabolites, six pentacyclic oxindole alkaloids, speciophylline, mitraphylline, pteropodine, uncarine $\mathrm{F}$, isopteropodine and isomitraphylline, are considered to be the biochemical markers and are used to standardize commercial herbal medicines. The present study describes the development of an analytical methodology to determine the profile of these alkaloid markers through thin layer chromatography (TLC). This development has also included a comparison among the use of the crude methanol extract and fractions obtained through the classical acid-base partition or by using the basic resin Polyamide 6. Silica gel was used as stationary phase with the variation of some parameters such as solvent systems, sample concentration, distance of development and detection method. The TLC method developed was shown to be reliable, reproducible and selective for the target alkaloids. It has been applied to the analysis of leaves and stems from both species as well as phytopharmaceutical derivatives based on $U$. tomentosa.
\end{abstract}

Keywords: Uncaria tomentosa, Uncaria guianensis, Rubiaceae, oxindole alkaloids, thin layer chromatography. 


\section{INTRODUÇÃO}

O gênero Uncaria (Rubiaceae), típico de regiões tropicais, é representado na América do Sul e Central por duas espécies (Ridscale, 1978): U. tomentosa (Willd.) DC. e U. guianensis (Aubl.) Gmel., conhecidas popularmente como unha-de-gato. Ambas são trepadeiras perenes, raramente cultivadas, possuindo diferenças anatômicas que facilmente as distinguem entre si (Vilches, 1997; Revilla, 2002). Possuem uma longa história de usos similares na medicina popular, principalmente no tratamento do câncer, na cura de feridas, alergias e artrites (Vilches, 1997; Revilla, 2002). A espécie $U$. guianensis tem se sobressaído por sua comprovada atividade antiinflamatória e antioxidante. Já a espécie $U$. tomentosa, mais estudada, destaca-se por sua atividade imunoestimulante, sendo também citotóxica, antiinflamatória e antioxidante (Heitzman et al., 2005). Em função de sua propriedade imunoestimulante, $U$. tomentosa é atualmente uma planta de alto valor comercial no Brasil e no mundo. As cascas do caule e as folhas da espécie são comercializadas in natura ou como fitoterápicos na forma de cápsula ou comprimido: partes da planta secas e moídas ou extrato etanólico seco. Ambas as espécies contêm, embora em proporções diferentes, alcalóides oxindólicos, $N$-oxi-oxindólicos e indólicos, triterpenos glicosilados, taninos e flavonóides. Testes farmacológicos in vitro e in vivo demonstraram que os alcalóides oxindólicos pentacíclicos presentes em U. tomentosa (Figura 1) eram os principais responsáveis por sua propriedade imunoestimulante (Heitzman et al., 2005) e atualmente, eles são usados para aferir a qualidade da espécie. A concentração desses alcalóides pode variar nas diferentes partes da planta e sazonalmente (Laus et al., 1997). É possível encontrar também um quimiotipo que contém preferencialmente alcalóides oxindólicos tetracíclicos (que não possuem atividade imunoestimulante). Esses alcalóides podem eventualmente co-ocorrer com os pentacíclicos (Laus et al., 1997). Em U. guianensis, de um modo geral, a concentração de alcalóides oxindólicos é bem menor do que em U. tomentosa (Laus; Keplinger, 2003).

Devido ao grande interesse comercial dessas plantas, é fundamental o desenvolvimento de métodos analíticos que possam monitorar a qualidade do material vegetal e dos fitoterápicos derivados. O presente trabalho descreve o desenvolvimento e a aplicação de metodologia de análise dos seis principais alcalóides oxindólicos pentacíclicos das espécies Uncaria tomentosa $e U$. guianensis por cromatografia em camada delgada.

A cromatografia em camada delgada (CCD) é um método rápido, eficiente, de baixo custo e largamente empregado em controle de qualidade de plantas medicinais, tanto em matéria-prima vegetal quanto em fitoterápicos derivados (Julião et al., 2003). Embora sejam encontrados na literatura alguns métodos para análise por CCD de alcalóides oxindólicos em espécies do gênero Uncaria (Philipson; Hemingway, 1975; Ginkel, 1996; Wagner; Bladt, 1996; Keplinger et al., 2002; Glensk et al., 2004) estes ou não são descritos com detalhes e/ou os alcalóides oxindólicos pentacíclicos não são identificados. Em função da complexidade da composição dos extratos alcoólicos brutos de ambas as espécies, que incluem grande quantidade de taninos (complexantes dos alcalóides), especialmente em amostras provenientes de caule ou casca de caule, a maioria das análises dos alcalóides presentes tem sido feita ou pelo pré-tratamento com base (hidróxido de amônio) do material vegetal seguido de extração com solvente (Ginkel, 1996) e/ou pela submissão, após extração, à metodologias que conduzem à frações enriquecidas desses alcalóides (Ganzera et al., 2001; Philipson; Hemingway, 1975; Wagner; Bladt, 1996). No entanto não há até o momento a descrição detalhada de uma metodologia para a determinação por CCD do perfil<smiles>COC(=O)[C@]1(C)CO[C@H](C)[C@H]2CN3CC[C@]2([C@H]2C(=O)Nc4ccccc42)C[C@@H]31</smiles>

1- Especiofilina

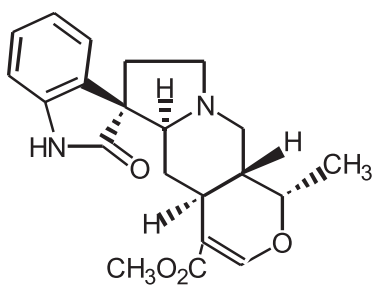

4- Isomitrafilina<smiles>COC(=O)[C@]1(C)CO[C@H](C)[C@@H]2CN3CC[C@]4(C(=O)Nc5ccccc54)[C@H]3C[C@@H]21</smiles>

2- Mitrafilina<smiles>COC(=O)[C@]1(C)CO[C@H](C)[C@@H]2CN3CC[C@]4(C(=O)Nc5ccccc54)[C@H]3C[C@@H]21</smiles>

5- Pteropodina

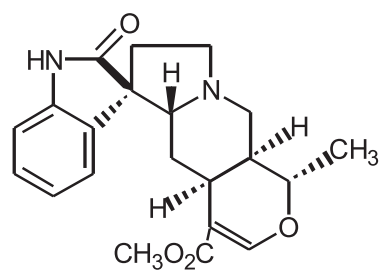

3- Uncarina $\mathrm{F}$

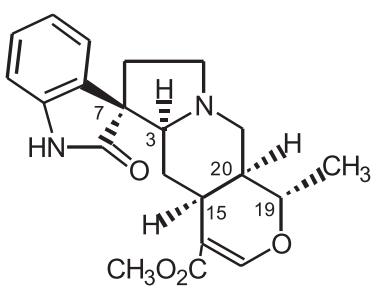

6- Isopteropodina

Figura 1. Estrutura dos seis alcalóides oxindólicos pentacíclicos considerados como marcadores das espécies sul-americanas de Uncaria 
dos alcalóides oxindólicos pentacíclicos nas espécies U. tomentosa e U. guianensis. No presente trabalho foram analisadas algumas condições cromatográficas descritas na literatura, otimizando-se vários parâmetros e introduzindo procedimentos, com o objetivo de desenvolver um método simples e reprodutível que pudesse detectar com precisão os alcalóides marcadores presentes na(s) amostra(s) avaliada(s). Como parte do desenvolvimento desta metodologia, foram comparados os perfis cromatográficos em CCD dos alcalóides alvos tanto pelo uso de extrato metanólico bruto quanto de frações enriquecidas desses alcalóides obtidas pela submissão do extrato bruto à partição ácido-base clássica e a tratamento com resina básica Poliamida 6 .

\section{MATERIAL E MÉTODOS}

\section{Desenvolvimento do método em CCD}

No estabelecimento da metodologia por CCD foi utilizada uma amostra de referência, não comercial, constituída de uma fração enriquecida nos alcalóides oxindólicos marcadores das espécies, obtida por partição ácido-base clássica a partir do extrato etanólico das cascas do caule de $U$. tomentosa previamente secas e moídas, coletadas em Cruzeiro do Sul, Acre (dados sobre identificação e exsicata em Miranda et al., 2003) e fornecidas pela empresa Biosapiens. Essa fração foi submetida à cromatografia líquida de alta eficiência (CLAE) com detector de ultravioleta (UV) a 245 $\mathrm{nm}$, com caracterização dos alcalóides oxindólicos pentacíclicos marcadores por seus tempos de retenção em condições descritas na literatura (Laus; Keplinger, 1994) e por CLAE acoplada a detector por photodiode array e espectrometria de massas (CLAE-DAD-EM) com monitoramento do íon $[\mathrm{M}+1]^{+}$em $\mathrm{m} / z 369$ e análise dos espectros de UV (Mazzei, 2004; Lopez-Avila et al., 1997). Para obtenção das substâncias padrão, a fração foi refluxada com éter metil $t$-butílico levando à precipitação, após resfriamento, de mitrafilina (2) que foi posteriormente purificada por cristalização nesse mesmo solvente. O sobrenadante foi seco a pressão reduzida e submetido seqüencialmente à cromatografia em coluna (CC) em gel de sílica com gradiente de solvente hexano-acetato de etila-metanol e à CLAE em fase reversa para isolamento de pteropodina (5) e isopteropodina (6) (Laus; Keplinger, 1994; Mazzei et al., 2002). As substâncias puras foram identificadas estruturalmente por espectrometria na região do infravermelho (IV), técnicas de ressonância magnética nuclear de hidrogênio e carbono-13 (RMN ${ }^{1} \mathrm{H}$ $\left.\mathrm{e}^{13} \mathrm{C}\right)\left(300 \mathrm{MHz}\right.$ e $75 \mathrm{MHz}$ respectivamente, $\mathrm{CDCl}_{3}$, TMS como padrão interno) em uma e duas dimensões e análise dos espectros de UV e EM em CLAE-DAD-EM e seus dados comparados àqueles disponíveis na literatura (Seki et al., 1993; Lopez-Avila et al., 1997). A determinação da pureza foi feita por integração dos cromatogramas de íons totais em CLAE-EM.

Mitrafilina (1): sólido branco cristalino; CLAEDAD-EM: $\lambda_{\max } 244 \mathrm{~nm}, \mathrm{~m} / z 369[\mathrm{M}+1]^{+}, \mathrm{t}_{\mathrm{R}} 3,86 \mathrm{~min}$ (pureza $>95 \%$ ); $\mathrm{RMN}{ }^{1} \mathrm{H}: 1,11(3 \mathrm{H}, \mathrm{d}, J 6,6 \mathrm{~Hz}, \mathrm{H}-18)$, $2,11(2 \mathrm{H}, \mathrm{m}, \mathrm{H}-15$ e 20$), 2,42(1 \mathrm{H}, \mathrm{dd}, J 2,6,10,1 \mathrm{~Hz}$, $\mathrm{H}-3), 3,60\left(3 \mathrm{H}, \mathrm{s}, \mathrm{CH}_{3} \mathrm{O}\right), 4,37$ (1H, qd, $J 3,0,6,6 \mathrm{~Hz}, \mathrm{H}-$ 19), 7,43 (1H, s, H-17), 7,91 (1H, s, NH); RMN ${ }^{13} \mathrm{C}: 15,1$ (C-18), 50,9 ( $\left.\mathrm{CH}_{3} \mathrm{O}\right), 55,7$ (C-7), 74,0 (C-19), 74,8 (C-3), 107,1 (C-16), 114,9 (C-12), 122,8 (C-10), 123,2 (C-9), 154,3 (C-17), 167,3 (C-22), 181,2 (C-2); IV (KBr) $v_{\text {max }}$ 1622, 1705, 1725; 3269, $3412 \mathrm{~cm}^{-1}$.

Pteropodina (5): sólido branco cristalino; CLAE-DAD-EM: $\lambda_{\text {max }} 247 \mathrm{~nm}, m / z 369[\mathrm{M}+1]^{+}, \mathrm{t}_{\mathrm{R}} 4,82$ $\min$ (pureza 99\%); RMN ${ }^{1} \mathrm{H}: 1,41(3 \mathrm{H}, \mathrm{d}, J 6,2 \mathrm{~Hz}, \mathrm{H}-18)$, $1,58(1 \mathrm{H}, \mathrm{m}, \mathrm{H}-20), 2,44$ (1H, ddd, $J 4,4,4,7,11,5 \mathrm{~Hz}, \mathrm{H}-$ $15), 3,60\left(3 \mathrm{H}, \mathrm{s}, \mathrm{CH}_{3} \mathrm{O}\right), 4,56(1 \mathrm{H}, \mathrm{qd}, J 6,2,10,6 \mathrm{~Hz}, \mathrm{H}-$ 19), 7,49 (1H, s, H-17), 7,93 (1H, sl, NH); IV (KBr) $v_{\max }$ $1624,1701,3257 \mathrm{~cm}^{-1}$.

Isopteropodina (6): sólido branco cristalino; CLAE-DAD-EM: $247 \mathrm{~nm}, \mathrm{~m} / z 369[\mathrm{M}+1]^{+}, \mathrm{t}_{\mathrm{R}}$ 7,49 min (pureza 100\%); RMN ${ }^{1} \mathrm{H}: 1,41(3 \mathrm{H}, \mathrm{d}, J 6,2 \mathrm{~Hz}, \mathrm{H}-18$ ), 1,59 (1H, m, H-20), 2,57 (1H, dd, J 2,8, 11,7 Hz, H-3), $3,60\left(3 \mathrm{H}, \mathrm{s}, \mathrm{CH}_{3} \mathrm{O}\right), 4,35$ (1H, qd, $J$ 6,2, 10,8 Hz, H-19), $7,41(1 \mathrm{H}, \mathrm{s}, \mathrm{H}-17), 8,80(1 \mathrm{H}, \mathrm{s}, \mathrm{NH})$; $\mathrm{RMN}{ }^{13} \mathrm{C}: 18,8(\mathrm{C}-$ 18), 51, $2\left(\mathrm{OCH}_{3}\right), 71,4$ (C-3), 124,7 (C-9), 127,8, 140,5 (C-13), 167,8 (C-22), 181,6 (C-2); IV (KBr) v $v_{\max } 1633$, $1679,1700,1719,3247 \mathrm{~cm}^{-1}$.

As análises em CCD foram desenvolvidas em placas pré-elaboradas de gel de sílica (cromatofolha Merck, gel de sílica $60 \mathrm{~F}_{254}, 0,2 \mathrm{~mm}$ ). As amostras foram aplicadas manualmente, utilizando-se microseringa de 25 $\mu \mathrm{L}$ (Hamilton ref. 80465).

Vários parâmetros foram ensaiados: largura da aplicação da amostra, espaço de eluição, tipos de reveladores, concentração da amostra e sistemas de fase móvel. Como primeira etapa do processo, foram testados quatro sistemas de solvente como fase móvel, dentre aqueles descritos na literatura (Keplinger et al., 2002; Philipson; Hemingway, 1975) que aliavam o maior potencial de separação dos alcalóides pentacíclicos em questão com a facilidade de manuseio: acetato de etila/ hexano (95:5), clorofórmio/acetona (5:4), clorofórmio/ metanol (95:5), acetato de etila/isopropanol/hidróxido de amônio (16:3:1). Depois de escolhido o sistema de eluente, testou-se a distância ótima de desenvolvimento. Para tal, foram utilizadas placas cromatográficas com $11,5,15$ e $20 \mathrm{~cm}$ de comprimento, com espaço de corrida de $10,13,5$ e $18,5 \mathrm{~cm}$ respectivamente. Para o teste da concentração mínima de detecção, e conseqüentemente da concentração ideal de análise, preparou-se uma solução de $5 \mathrm{mg} / \mathrm{mL}$ da amostra de referência em metanol. Dessa solução, foram aplicadas alíquotas de 6, 10, 16, 20, 26, 30 e $36 \mu \mathrm{L}$. Em seguida, a largura das bandas de aplicação da amostra foi ensaiada com 0,5 e $1 \mathrm{~cm}$. A visualização das manchas foi efetuada por exposição à luz UV a $254 \mathrm{~nm}$ e a revelação, através de borrifamento com reagente de Dragendorff/nitrito de sódio 10\% (Wagner; Bladt, 1996). As placas foram documentadas por fotografia digital. 
Identificação dos alcalóides na placa cromatográfica

Os seis alcalóides marcadores foram identificados na placa cromatográfica pela comparação dos $R_{f}$ das manchas na amostra de referência com os $R_{f}$ de padrões isolados e identificados estruturalmente [mitrafilina (2), pteropodina (5) e isopteropodina (6)], conforme descrito anteriormente, e por intermédio da comparação com a ordem de eluição descrita por Keplinger et al. (2002). Para os padrões, foram aplicados $10 \mu \mathrm{L}$ de uma solução de $1 \mathrm{mg} / \mathrm{mL}$.

\section{Métodos de extração de alcalóides}

Para comparar os diferentes métodos de extração de alcalóides utilizou-se a mesma partida de cascas de caule de U. tomentosa, coletada em Cruzeiro do Sul, Acre, utilizada na obtenção da amostra de referência e no isolamento das substâncias padrão. Os extratos foram preparados segundo metodologia otimizada por Ganzera et al. (2001), onde $750 \mathrm{mg}$ do material vegetal seco e moído foram extraídos com $2,5 \mathrm{~mL}$ de metanol com sonicação por 10 min seguido de centrifugação por 5 min. O processo foi repetido 4 vezes e os sobrenadantes reunidos. No tratamento com resina básica (Ganzera et al., 2001) os sobrenadantes foram transferidos para balão volumétrico de $10 \mathrm{~mL}$ e o volume completado com metanol. Em seguida foi retirada uma alíquota de $3 \mathrm{~mL}$ e adicionado a esta $300 \mathrm{mg}$ de resina Nylon 6 Radilon S natural (Radici Plastics Ltda.) (Poliamida 6) de granulometria 53-125 $\mu \mathrm{m}$ e a mistura mantida sob agitação magnética por $15 \mathrm{~min}$. O sobrenadante foi então seco a pressão reduzida e pesado. Na partição ácidobase, os sobrenadantes resultantes da extração depois de reunidos foram secos a pressão reduzida e pesados. $\mathrm{O}$ extrato seco foi tratado com solução de ácido clorídrico $0,1 \mathrm{~N}$ em uma relação $0,15 \mathrm{~mL} / \mathrm{mg}$. A mistura foi colocada no banho de ultra-som por 5 min e em seguida extraída com acetato de etila, sendo o volume de acetato de etila utilizado igual ao do ácido clorídrico (3 vezes). A fração aquosa foi alcalinizada com hidróxido de amônio até $\mathrm{pH}$ 9-10 e extraída com o mesmo volume de acetato de etila (4 vezes), gerando uma fração orgânica enriquecida em alcalóides. A fração foi seca com sulfato de sódio anidro, filtrada, evaporada à pressão reduzida e pesada. As amostras foram aplicadas nas placas em duplicata.

\section{Aplicação da metodologia desenvolvida}

O método desenvolvido foi aplicado na análise de amostras de folhas e cascas de caule de $U$. guianensis, coletadas em Juruema, Mato Grosso e doadas pela O.N.G. Pró-Natura (a planta foi identificada pelo botânico Pierro Delprete do New York Botanic Garden e uma exsicata está depositada no Herbário Central da Universidade Federal de Mato Grosso, com o $\mathrm{n}^{\circ}$ 24.715), e em amostras de folhas e cascas de caule de U. tomentosa, coletadas no Acre e doadas pela EmbrapaAcre (dados sobre identificação e exsicata em Miranda et al., 2003). O método foi aplicado também na análise de quatro fitoterápicos à base de $U$. tomentosa de diferentes fornecedores, aleatoriamente adquiridos no comércio local. A descrição dos fitoterápicos analisados e de seus respectivos procedimentos de extração encontra-se no Quadro 1. As amostras foram aplicadas nas placas em duplicata.

\section{RESULTADOS}

A largura de aplicação de $1 \mathrm{~cm}$ proporcionou uma melhor resolução das bandas dos alcalóides em relação à de $0,5 \mathrm{~cm}$. A distância de desenvolvimento de $10 \mathrm{~cm}$ foi a escolhida por proporcionar uma separação satisfatória dos alcalóides, conjugado à praticidade e menor custo. Os perfis obtidos para os seis alcalóides

Quadro 1. Descrição dos fitoterápicos à base de Uncaria tomentosa analisados e de seus respectivos procedimentos de extração

\begin{tabular}{|c|c|c|c|}
\hline Fitoterápico & Forma farmacêutica & $\begin{array}{c}\text { Composição e parte da planta } \\
\text { utilizada* }\end{array}$ & Procedimento de extração** \\
\hline A & cápsula gelatinosa dura & $\begin{array}{c}\text { casca em pó } \\
(410 \mathrm{mg} / \text { cápsula })\end{array}$ & $\begin{array}{c}\text { abertura manual da cápsula e } \\
\text { extração de seu conteúdo }(747 \\
\mathrm{mg} / 10 \mathrm{~mL})\end{array}$ \\
\hline $\mathrm{B}$ & comprimido & $\begin{array}{c}\text { Parte da planta não informada e } \\
\text { excipientes }(190 \mathrm{mg} \text { de } \\
\text { planta/comprimido) }\end{array}$ & $\begin{array}{c}\text { trituração do comprimido seguida } \\
\text { de extração } \\
(1621 \mathrm{mg} / 10 \mathrm{~mL})\end{array}$ \\
\hline C & comprimido & $\begin{array}{c}\text { extrato seco do córtex da raiz e } \\
\text { excipientes }(100 \mathrm{mg} \text { de } \\
\text { extrato/comprimido) }\end{array}$ & $\begin{array}{c}\text { trituração do comprimido seguida } \\
\text { de extração } \\
(638 \mathrm{mg} / 10 \mathrm{~mL})\end{array}$ \\
\hline $\mathrm{D}$ & cápsula gelatinosa dura & $\begin{array}{c}\text { folha em pó } \\
(330 \mathrm{mg} / \text { cápsula) }\end{array}$ & $\begin{array}{c}\text { abertura manual da cápsula e } \\
\text { extração de seu conteúdo } \\
(754 \mathrm{mg} / 10 \mathrm{~mL})\end{array}$ \\
\hline
\end{tabular}

*conforme informado pelo fabricante; **todas as extrações foram feitas com MeOH. 


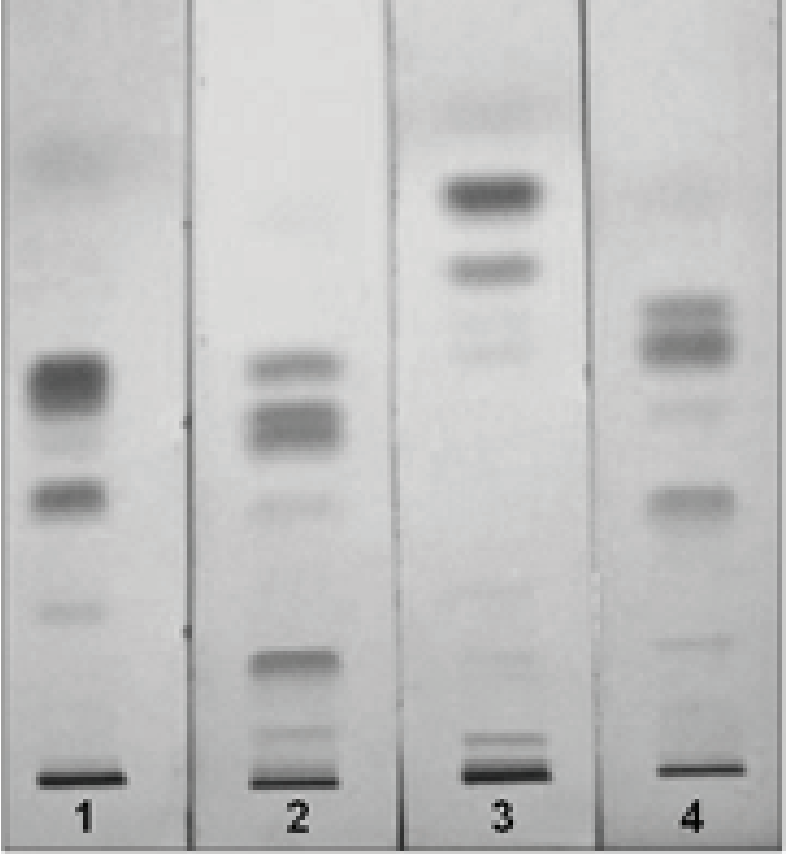

1: $\mathrm{CHCl}_{3} / \mathrm{MeOH}$ 95:5; 2:Hexano/AcOEt 5:95; 3:AcOEt/iPrOH/ $\mathrm{NH}_{4} \mathrm{OH}(16: 3: 1) ; 4: \mathrm{CHCl}_{3} /$ Acetona 5:4. Fase estacionária: gel de sílica; visualização: UV, $254 \mathrm{~nm}$; dist. desenvolvimento: 10 $\mathrm{cm}$; conc. amostra de referência: $5 \mathrm{mg} / \mathrm{mL}$; vol. aplicado: 10 $\mu \mathrm{L}$.

Figura 2. Perfil dos alcalóides oxindólicos da amostra de referência nos quatro sistemas de fase móvel testados.

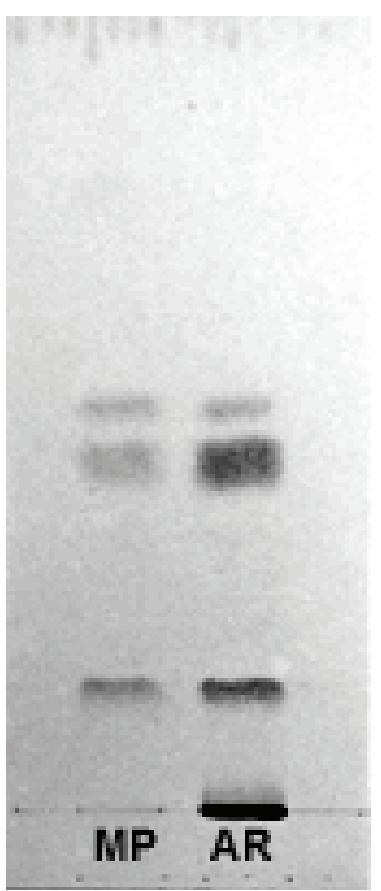

MP: Mistura de Padrões; AR: Amostra de Referência. Fase móvel: Hexano/AcOEt 5:95; fase estacionária: gel de sílica; dist. desenvolvimento: $10 \mathrm{~cm}$; conc. amostra de referência: 5 $\mathrm{mg} / \mathrm{mL}$; conc. padrões: $1 \mathrm{mg} / \mathrm{mL}$; vol. aplicado: $10 \mu \mathrm{L}$.

Figura 4. Revelação da amostra de referência por borrifamento com reagente de Dragendorff/nitrito de sódio $10 \%$.

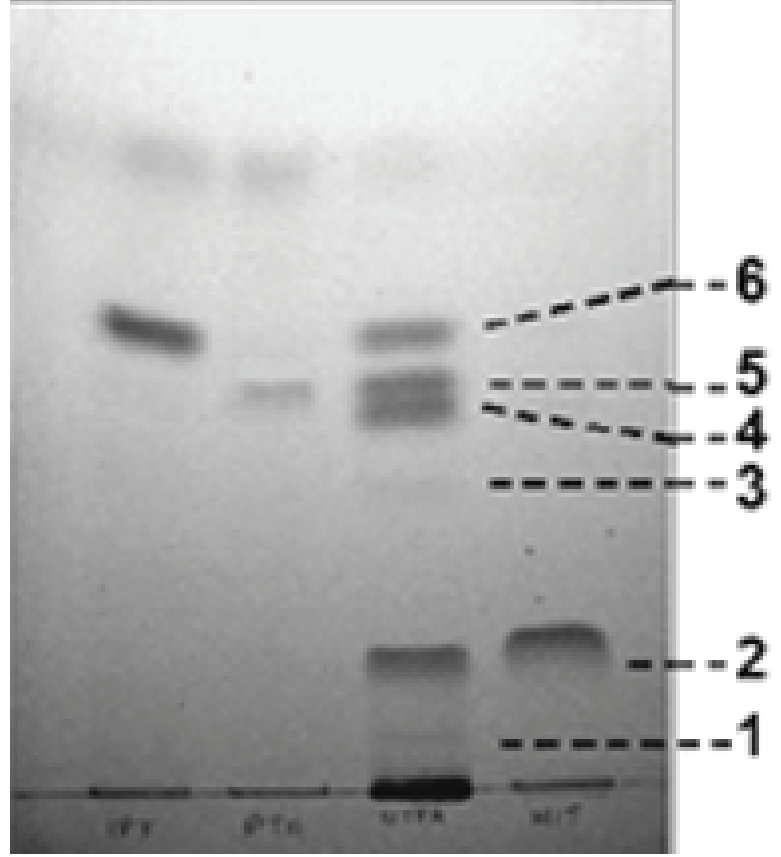

1-Especiofilina, 2-Mitrafilina, 3-Uncarina F, 4-Isomitrafilina, 5-Pteropodina, 6-Isopteropodina. Fase móvel: Hexano/AcOEt 5:95; fase estacionária: gel de sílica; visualização: UV, $254 \mathrm{~nm}$; dist. desenvolvimento: $10 \mathrm{~cm}$; conc. amostra de referência: $5 \mathrm{mg}$ / $\mathrm{mL}$; conc. padrões: $1 \mathrm{mg} / \mathrm{mL}$; vol. aplicado: $10 \mu \mathrm{L}$.

Figura 3. Identificação dos alcalóides oxindólicos presentes na amostra de referência.

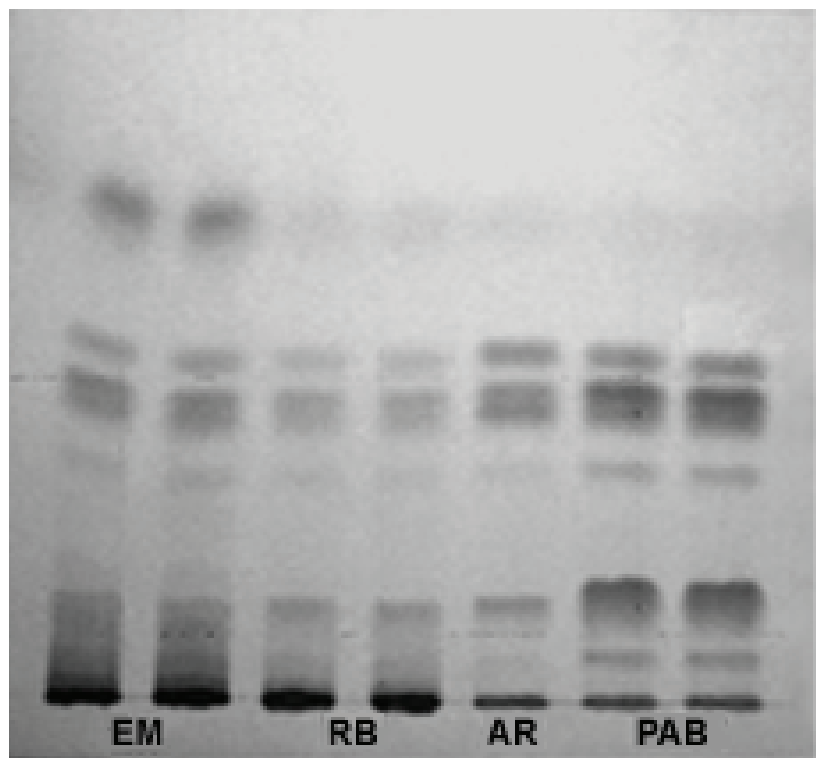

EM: Extrato $\mathrm{MeOH}$ bruto; RB: Extrato $\mathrm{MeOH}$ tratado com resina básica Poliamida 6; AR: Amostra de Referência; PAB: Extrato $\mathrm{MeOH}$ submetido à partição ácido-base. Fase móvel: Hexano/ AcOEt 5:95; fase estacionária: gel de sílica; visualização: UV, 254 $\mathrm{nm}$; dist. desenvolvimento: $10 \mathrm{~cm}$; conc. amostra de referência e de PAB: $5 \mathrm{mg} / \mathrm{mL}$; conc. extratos: $50 \mathrm{mg} / \mathrm{mL}$; vol. aplicado: 10 $\mu \mathrm{L}$.

Figura 5. Comparação entre os perfis de alcalóides de uma amostra de $U$. tomentosa em relação a diferentes métodos de extração. 
Tabela 1. Faixa de valores de $\mathrm{R}_{\mathrm{f}}$ dos alcalóides oxindólicos marcadores

\begin{tabular}{lcc}
\hline & Alcalóide & Faixa de valores de $\mathrm{R}_{\mathrm{f}}{ }^{*}$ \\
\hline Especiofilina (1) & $0,05-0,06$ \\
Mitrafilina (2) & $0,15-0,16$ \\
Uncarina F (3) & $0,34-0,40$ \\
Isomitrafilina (4) & $0,44-0,49$ \\
Pteropodina (5) & $0,47-0,53$ \\
Isopteropodina (6) & $0,53-0,60$ \\
\hline
\end{tabular}

*valores determinados a partir do cromatograma da amostra de referência (AR) nas diversas placas cromatográficas apresentadas. Fase móvel: Hexano/AcOEt 5:95; fase estacionária: gel de sílica; dist. desenvolvimento: $10 \mathrm{~cm}$.

nos quatro sistemas de eluentes testados, utilizandose uma largura de aplicação de amostra e distância de desenvolvimento respectivamente de 1 e $10 \mathrm{~cm}$ (placa cromatográfica de $11,5 \mathrm{~cm}$ ), são mostrados na Figura 2. O sistema de fase móvel escolhido foi o que utiliza acetato de etila/hexano 95:5 por apresentar melhor separação dos alcalóides alvos. A concentração ótima de análise para essa fração enriquecida foi a que utilizou uma alíquota de $10 \mu \mathrm{L}$ da solução estoque de $5 \mathrm{mg} / \mathrm{mL}$. Esta foi a menor concentração que permitiu a visualização de todos os alcalóides presentes, levando-se em conta a variação das concentrações dos mesmos, com substâncias amplamente majoritárias e outras ausentes, ou em concentração muito baixa.

A identificação dos seis alcalóides marcadores na placa cromatográfica é mostrada na Figura 3. A visualização sob lâmpada de UV a 254 nm (Figuras 2 e 3) mostrou-se mais prática que a revelação por borrifamento com reagente de Dragendorff/nitrito de sódio 10\%, que apresentou manchas amarelo-amarronzadas instáveis (Figura 3) (em todas as figuras a seguir as placas cromatográficas mostram a visualização por irradiação em UV a $254 \mathrm{~nm})$.

A comparação entre os perfis de alcalóides obtidos pelo uso do extrato metanólico bruto e frações enriquecidas de alcalóides, oriundas do tratamento do extrato com resina Poliamida 6 e da partição ácido-base, é mostrada na Figura 5.

As Figuras 6 e 7 mostram a aplicação da metodologia desenvolvida na análise de quatro fitoterápicos comerciais à base de $U$. tomentosa de diferentes fabricantes assim como na análise de amostras

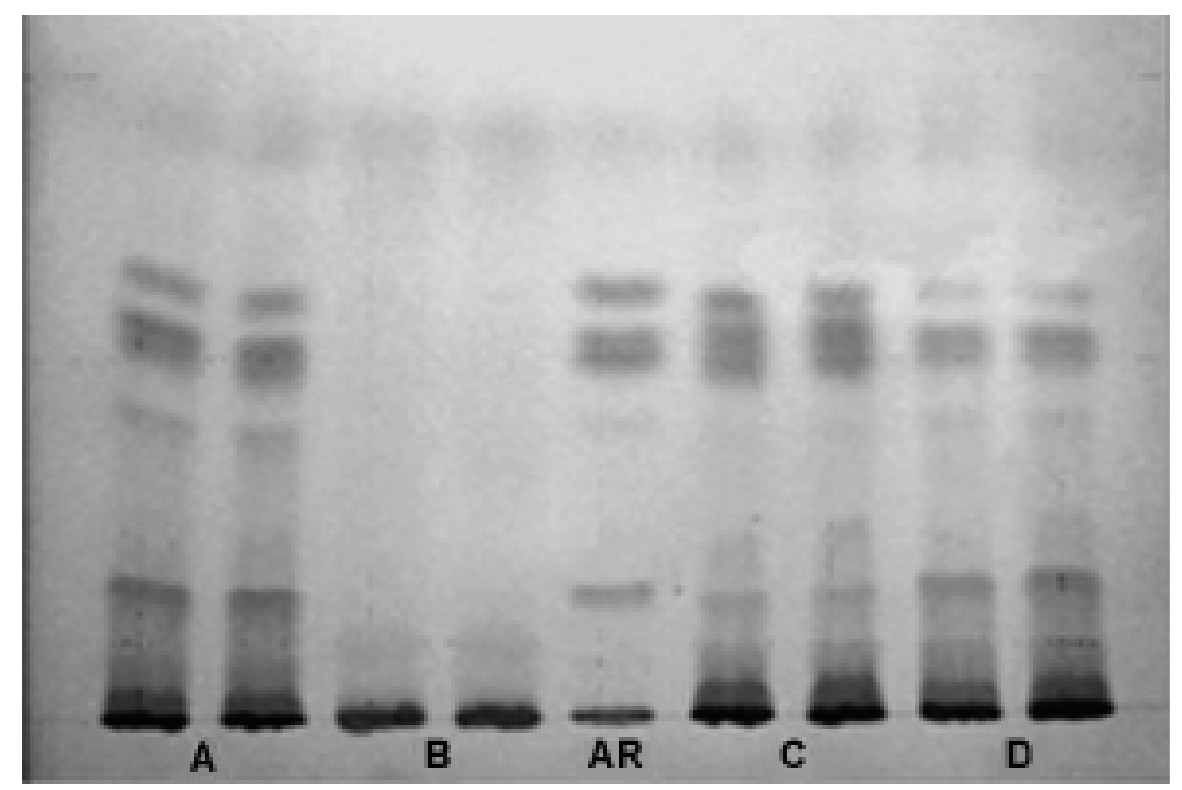

A,B,C,D: ver Quadro 1; AR: Amostra de Referência. Fase móvel: Hexano/AcOEt 5:95; fase estacionária: gel de sílica; visualização: UV, $254 \mathrm{~nm}$; dist. desenvolvimento: $10 \mathrm{~cm}$; conc. amostra de referência: $5 \mathrm{mg} / \mathrm{mL}$; conc. amostras de fitoterápicos: $50 \mathrm{mg} / \mathrm{mL}$; vol. aplicado: $10 \mu \mathrm{L}$.

Figura 6. Perfil dos alcalóides em amostras de fitoterápicos comerciais à base de $U$. tomentosa. 


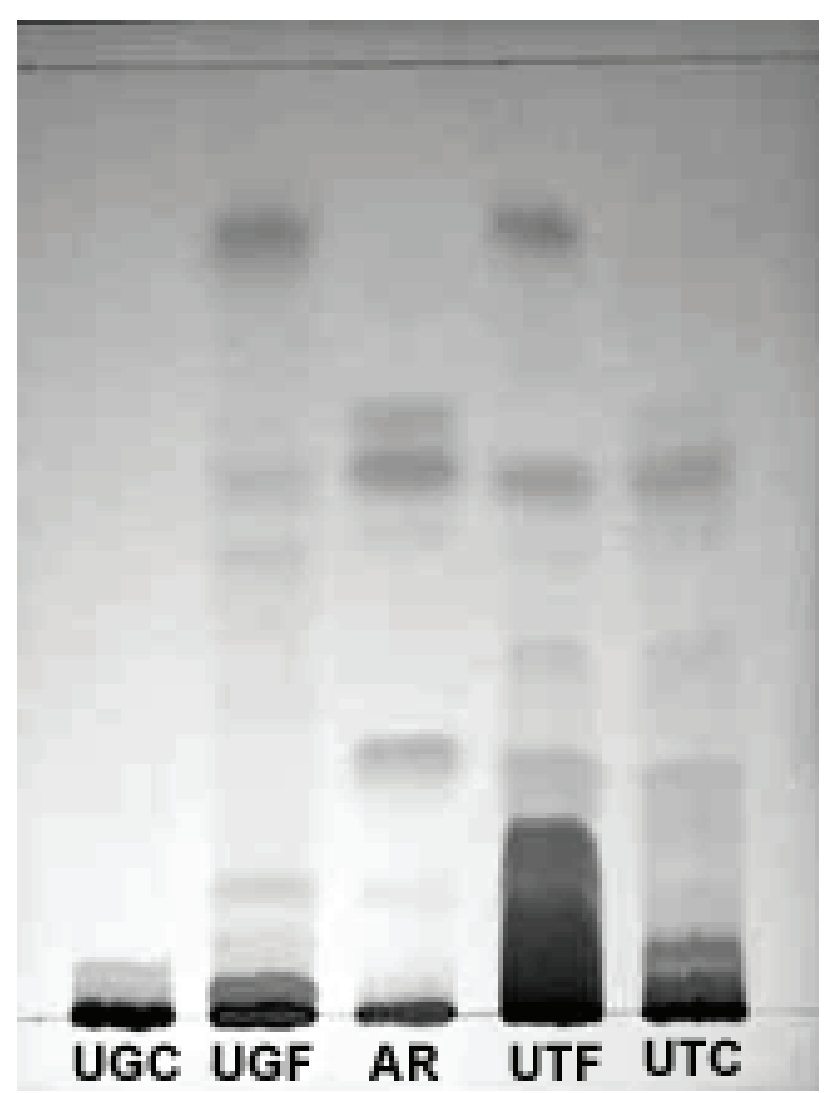

UGC e UGF: respectivamente caule e folha de U. guianensis; UTC e UTF: respectivamente caule e folha de $U$. tomentosa; AR: Amostra de Referência. Fase móvel: Hexano/AcOEt 5:95; fase estacionária: gel de sílica; visualização: UV, $254 \mathrm{~nm}$; dist. desenvolvimento: $10 \mathrm{~cm}$; conc. amostra de referência: $5 \mathrm{mg} /$ $\mathrm{mL}$; conc. amostras: $50 \mathrm{mg} / \mathrm{mL}$; vol. aplicado: $10 \mu \mathrm{L}$.

Figura 7. Perfis dos alcalóides em amostras de U. tomentosa e U. guianensis

de folhas e cascas de caule das duas espécies.

A faixa dos valores de $\mathrm{R}_{\mathrm{f}}$ para os alcalóides alvos está descrita na Tabela 1.

\section{DISCUSSÃO}

As pequenas variações que ocorreram nos $R_{f}$ de algumas substâncias (Tabela 1) devem-se possivelmente às oscilações na saturação da câmara cromatográfica.

A comparação dos cromatogramas obtidos com os dois métodos de extração testados frente ao cromatograma do extrato bruto (Figura 5), revela que a melhor visualização dos alcalóides foi alcançada quando se utilizou a fração enriquecida pela partição ácido-base (PAB). Já o cromatograma do extrato bruto revelou muitas impurezas na base, dificultando, ou mesmo impedindo a visualização dos alcalóides de menor $\mathrm{R}_{\mathrm{f}}$ [especiofilina (1) e mitrafilina (2)]. Por outro lado, o tratamento do extrato bruto com a resina básica Poliamida 6 (RB), cuja função é a de reter os taninos, proporcionou uma visualização muito satisfatória dos alcalóides. Para se obter esses resultados, foram utilizados, tanto para o extrato bruto quanto para o semi-purificado por resina básica, $10 \mu \mathrm{L}$ de uma solução $50 \mathrm{mg} / \mathrm{mL}$ (500 $\mu$ g aplicados) e para a fração obtida por partição ácido-base as mesmas condições da amostra de referência (50 $\mu \mathrm{g}$ aplicados). A concentração dez vezes maior para o extrato bruto e para o semipurificado baseou-se no rendimento aproximado de 10\% da fração enriquecida em alcalóides, obtida por partição ácido-base, em relação ao extrato bruto. Pode-se concluir que todos os métodos de extração apresentam vantagens e desvantagens, quer pelo custo, quer pelo grande número de etapas ou pela menor eficiência de visualização das manchas dos alcalóides alvo, e que a escolha pode ser feita em termos de número de amostras, recursos disponíveis e objetivos da análise. O método de extração com resina foi o escolhido na análise dos fitoterápicos (Figura 6). Nesse caso, aliam-se a praticidade da extração em fase sólida e o objetivo de se efetuar uma análise comparativa mais detalhada entre os diversos fabricantes. Dessa forma pode-se constatar que o fitoterápico $\mathrm{B}$, ao contrário dos outros, não contém os alcalóides alvos. Para as amostras vegetais, a opção por aplicar o extrato bruto direto na placa cromatográfica (Figura 7), permitiu efetuar uma triagem preliminar rápida da presença e do perfil de alcalóides nesse material, observando-se a ausência e a aparente menor quantidade dos alcalóides oxindólicos pentacíclicos nas amostras de $U$. guianensis em relação às de $U$. tomentosa. Como constatado pelas diversas placas cromatográficas apresentadas, o método cromatográfico desenvolvido, independente da forma de extração dos alcalóides, mostrou-se confiável, reprodutivo e seletivo para os alcalóides oxindólicos pentacíclicos marcadores alvos, possibilitando uma análise qualitativa do perfil desses alcalóides nas amostras analisadas. Os resultados apresentados preenchem uma lacuna na literatura, e ao mesmo tempo disponibilizam um instrumento alternativo preciso e acessível para o controle de qualidade de produtos e drogas vegetais a partir de $U$. tomentosa e $U$. guianensis, ao validar a utilização de uma amostra de referência como sucedânea dos alcalóides oxindólicos pentacíclicos - marcadores químicos de custo elevado (Reif et al., 2004).

\section{AGRADECIMENTOS}

Os autores agradecem ao CNPq, CAPES e FUJB-UFRJ pelo auxílio financeiro ao projeto. A.C.S. e F.F.A. agradecem ao CNPq e G.M.B. e S.L.R. à FAPERJ pelas bolsas concedidas. Os autores agradecem também à Profa. Bluma G. Soares do IMA-UFRJ pela doação da resina Nylon 6, ao Prof. Marcos V. A. Fonseca e à Dra. Ma. Cristina L. F. P. Pinto do IQ-UFRJ pela moagem da mesma e ao Prof. Luiz Nelson L. F. Gomes do IQ-UFRJ pela fotografia das placas. Agradecem ainda ao Dr. Peter May, à O.N.G. Pro-Natura, à empresa Biosapiens, e ao Dr. João A. de Sousa da Embrapa-Acre pela doação do 
material vegetal utilizado no trabalho. E, finalmente, à Dra. Hélida B. N. Borges do Herbário Central da UFMT pelos dados da exsicata de $U$. guianensis.

\section{REFERÊNCIAS}

Ganzera M, Muhammad I, Khan RA, Khan IA 2001. Improved method for the determination of oxindole alkaloids in Uncaria tomentosa by high performance liquid chromatography. Planta Med 6: 447-450.

Ginkel A 1996. Identification of the alkaloids and flavonoids from Uncaria tomentosa bark by TLC in quality control. Phytother Res 10: S18-S19.

Glensk M, Źbikowska B, Cisowski W 2004. TLC separation of Uncaria tomentosa alkaloids on chemically modified stationary phases. J Planar Chromatogr 17: 14-17.

Heitzmam ME, Neto CC, Winiarz E, Vaisberg AJ, Hammond GB 2005. Ethnobotany, phytochemistry and pharmacology of Uncaria (Rubiaceae). Phytochemistry 66: 5-29.

Julião LS, Tavares ES, Lage CLS, Leitão SG 2003. Cromatografia em camada fina de extratos de três quimiotipos de Lippia alba (Mill) N.E.Br. (erva-cidreira). Rev Bras Farmacogn 13(Supl. 1): 36-38.

Keplinger K, Wurn M, Laus G 2002. Process and substances for the release of a growth-regulating factor from endothelial cells. USP 0039790 A1.

Laus G, Keplinger D 1994. Separation of stereoisomeric oxindole alkaloids from Uncaria tomentosa by high performance liquid chromatography. $J$ Chromatogr 662: 243-249.

Laus G, Brössner D, Keplinger K 1997. Alkaloids of Peruvian Uncaria tomentosa. Phytochemistry 45: 853-860.

Laus G, Keplinger K 2003. Alkaloids of Peruvian Uncaria guianensis (Rubiaceae). Phyton 43: 1-8.

Lopez-Avila V, Benedicto J, Robaugh D 1997. Supercritical fluid extraction of oxindole alkaloids from Uncaria tomentosa. J High Resol Chromatogr 20: 231-236.

Mazzei JL, Rosario SL, Silva RS, Siani AC, Valente LMM, D'Avila LA 2002. Scale-up of isolation of oxindole alkaloids from Uncaria tomentosa by HPLC using chromatographic model. Rev Fitoterapia 2: 289.

Mazzei JL 2004. Transposição em escala por modelos na produção de substâncias naturais por cromatografia líquida de alta eficiência. Rio de Janeiro, 227p. Tese de Doutorado - Escola de Química, Universidade Federal do Rio de Janeiro.

Miranda EM, Sousa JA, Pereira RCA 2003. Caracterização e avaliação de populações nativas de unha de gato [Uncaria tomentosa (Willd.) DC. e U. guianensis ( Aubl.) Gmel.] no vale do rio Juruá-AC. Rev Bras Pl Med 5: 41-46.

Philipson JD, Hemingway SR 1975. Chromatographic and spectroscopic methods for the identification of alkaloids from herbarium samples of the genus Uncaria. J Chromatogr 105: 163-178.

Reif K, Sievers H, Steffen J-P 2004. The role of chemical reference standards as analytical tools in the quality assessment of botanical materials - a European perspective. HerbalGram 63: 38-43.

Revilla J 2002. Plantas Úteis da Bacia Amazônica. v.II, Manaus: Instituto Nacional de Pesquisas da Amazônia/ SEBRAE-AM
Ridsdale CE 1978. A revision of Mitragyna and Uncaria (Rubiaceae). Blumea 24: 43-100.

Seki H, Takayama H, Aimi N, Sakai SI, Ponglux D 1993. A nuclear magnetic resonance study on the eleven stereoisomers of heteroyohimbine-type oxindole alkaloids. Chem Pharm Bull 41: 2077-2086.

Vilches LEO 1997. Uña de Gato. Género Uncaria. Estúdios Botánicos, Químicos y Farmacológicos de Uncaria tomentosa y Uncaria guianensis. $3^{\mathrm{a}}$. ed. Lima: Instituto de Fitoterapia Americano.

Wagner H, Bladt S 1996. Plant Drug Analysis: A Thin Layer Chromatography Atlas. Berlin: Springer. 